\title{
The brain decade in debate: II. Panic or anxiety? From animal models to a neurobiological basis
}

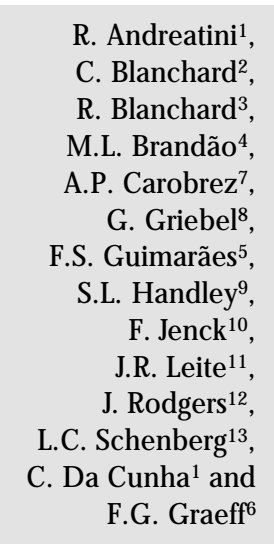

\section{Correspondence}

C. Da Cunha

Laboratório de Fisiologia e

Farmacologia do SNC

Departamento de Farmacologia,

UFPR

Caixa Postal 19.031

81531-990 Curitiba, PR

Brasil

E-mail: dacunha@bio.ufpr.br

Publication supported by FAPESP.

The names of the authors are

presented in alphabetic order,

except for the two last ones that

correspond to the organizer

(C. Da Cunha) of the Brain Decade

Series Symposia and to the

symposium chairman (F.G. Graeff).

Received August 15, 2000

Accepted January 3, 2001

\author{
'Laboratório de Fisiologia e Farmacologia do Sistema Nervoso Central, \\ Departamento de Farmacologia, Universidade Federal do Paraná, Curitiba, PR, Brasil \\ Departments of ${ }^{2}$ N eurobiology and ${ }^{3}$ Psychology, University of H awaii, Honolulu, HI, USA \\ ${ }^{4}$ Departamento de Psicobiologia, Faculdade de Filosofia Ciências e Letras de Ribeirão \\ Preto, ${ }^{5}$ Departamento de Fisiologia, Faculdade de Medicina de Ribeirão Preto, and \\ ${ }^{6}$ Departamento de Neuropsiquiatria, U niversidade de São Paulo, Ribeirão Preto, \\ SP, Brasil \\ ${ }^{7}$ Departamento de Farmacologia, U niversidade Federal de Santa Catarina, \\ Florianópolis, SC, Brasil \\ ${ }^{8}$ Sanofi Synthelabo, Central Nervous System Research Department, Bagneux, France \\ 9 Institute of Pharmaceutical Sciences, Aston University, Birmingham, England \\ ${ }^{10} \mathrm{H}$ offmann-La Roche Ltd., Basel, Switzerland \\ ${ }^{11}$ D epartamento de Psicologia, Escola Paulista de Medicina, \\ Universidade Federal de São Paulo, São Paulo, SP, Brasil \\ ${ }^{12}$ Ethopharmacology Laboratory, D epartment of Psychology, University of Leeds, \\ Leeds, England \\ ${ }^{13}$ Laboratório de Neurobiologia, Departamento de Ciências Fisiológicas, \\ Centro de Biomedicina, Universidade Federal do Espírito Santo, Vitória, ES, Brasil
}

\section{Abstract}

This article is a transcription of an electronic symposium sponsored by the Brazilian Society of Neuroscience and Behavior (SBNeC). Invited researchers from the European Union, North America and Brazil discussed two issues on anxiety, namely whether panic is a very intense anxiety or something else, and what aspects of clinical anxiety are reproduced by animal models. Concerning the first issue, most participants agreed that generalized anxiety and panic disorder are different on the basis of clinical manifestations, drug response and animal models. Also, underlying brain structures, neurotransmitter modulation and hormonal changes seem to involve important differences. It is also common knowledge that existing animal models generate different types of fear/anxiety. A challenge for future research is to establish a good correlation between animal models and nosological classification.

The last decade witnessed major advances in the understanding of the neuroanatomical and neurohumoral basis of anxiety. Also, pharmacological and psychotherapeutic treatment of anxiety disorders became more effective. Yet, some crucial issues concerning the neurobiological substrate of anxiety disorders remain open to debate. Among such questions two were selected for the following discussion, namely whether panic is a very intense anxiety or something else, and what aspects of clinical anxiety are repro-
Key words

- Panic

- Anxiety

- Fear

- Anxiolytics

- 5-HT

- Benzodiazepines

- Serotonin

- Amygdala

- Periaqueductal gray matter

- Animal models 
duced by animal models. The present article was based on the transcription of an electronic symposium sponsored by the Brazilian Society of Neuroscience and Behavior (SBNeC) held on April 26, 2000 at a chat site provided by the Conselho Nacional de Desenvolvimento Científico e Tecnológico (CNPq).

Frederico Graeff: Let us start with the first question: "Is panic a big anxiety or is it something else?"

Luiz Carlos Schenberg: Let me try. Concerning the question about anxiety and panic, maybe panic is a sudden and intense wave of anxiety; however, if so, this makes a big difference in terms of clinical, physiological and pharmacological aspects. Why such a big responsiveness?

Padua Carobrez: Recurrent panic sufferers can learn how to avoid places based on a general context cue, but do they learn how to cope with this in the future? They sure avoid places but the inability to learn during the attack does not allow them to challenge or face the situation in the future.

Sheila Handley: Professor Graeff has posed an interesting question. I think the answer to 'Is fear a big anxiety?' depends on what you include. Emotions are notoriously difficult to translate into words, and it would be very difficult to argue the question one way or the other on the basis of the emotion alone. But the picture may be different if inputs and outputs are included too. Back in 1962, Taylor (1) showed that the perceptual and stimulus properties of objects in the environment depend strongly on our cumulative experience of how we can interact with them. On this basis, one could argue that the stimulus properties giving rise to an emotion are an essential component of the emotional experience. The stimulus properties of cues to anxiety and to panic are arguably different and roughly comparable to distal and proximal cues, respectively (2). Behavioral and autonomic output and, cru- cially, the feedback we receive from these, may also be integral components. Yes shades of James-Lange - and also of Taylor's hypothesis, i.e., that our behavioral responses are important to how we view a stimulus. Intensity of the autonomic output arousal does seem to differ between anxiety and panic. Furthermore, panic attacks are frequently accompanied by an intense desire to flee/actual flight.

Padua Carobrez: Is anxiety a dysregulation of the normal defense system? How does panic fit into this?

Francisco Guimarães: Padua, panic is a normal defensive reaction. The big (and unsolved) question is why some people tend to develop 'spontaneous' panic attacks?

Caroline \& Robert Blanchard: Hi everybody! We're at home, with only one computer so our names will appear together. Is panic a big anxiety? Our response is no. Not clinically, not in terms of drug response, and certainly not in terms of animal models. We think the difference is that they represent exacerbation or abnormal functioning in two different sets of defense systems, flight for panic and risk assessment (and defensive threat/attack) for a more general anxiety. These are certainly not just our assumptions, and it is interesting that other tasks used to model panic and anxiety have generally been successful in calling specific attention to these particular behaviors.

Marcus Brandão: I agree with the Blanchards. I do not think that panic is a big generalized anxiety. There are several differences from the clinical viewpoint. Just to mention one, anxiety is an adaptive response with a slow onset while panic is sudden and does not serve any adaptive function. Besides there are some proposals, mainly Deakin and Graeff's (2), drawn from animal models of anxiety that consider them two distinct processes.

Luiz Carlos Schenberg: Marcus, of course panic is adaptive. Have you ever faced an angry pit bull? This is physiological 
panic. Pathological panic (anxiety neurosis) or anxiety are maladaptive, but not the physiological expression of both states.

Caroline \& Robert Blanchard: Schenberg asked an interesting question. Certainly the autonomic components associated with any high level defensive arousal will be useful in facing an angry pit bull. Some defensive behaviors will probably be more useful than others (flight is probably poor in that case). The question is what is useful and when, and the degree to which psychopathologies represent genuinely maladaptive defense both now and in the evolution of mammals.

Frederico Graeff: Recent results obtained by Cristina M. Del Ben with panic disorder patients in human models of anxiety show that they have high baseline anxiety, but normal levels of arousal (visual analog mood scale mental sedation factor). Simulated public speaking (seemingly related to innate fear) does not enhance anxiety in these patients or increase arousal. Yet they behave like controls in the conditioned fear test (skin conductance). These results are compatible with the suggestion that in panic patients high anxiety is inhibiting panic $(2,3)$.

Luiz Carlos Schenberg: We have almost completed a study on a group showing the reduction in defense reaction thresholds (in the dorsal periaqueductal gray matter) following dexamethasone administration. In this case, anxiety should facilitate panic.

John Rodgers: Whether panic is simply quantitatively different from generalized anxiety disorder is still an issue, certainly for clinicians (and as such, should also be so for preclinical researchers/animal modelers). A key line of evidence in favor of a qualitatively different phenomenon (leading to separate classification in DSM) was of course the apparently poor therapeutic response to benzodiazepines and good therapeutic response to monoamine oxidase inhibitors and tricyclics (and now also selective serotonin (5HT) reuptake inhibitors). However, antide- pressants are also very effective (some say more effective than benzodiazepines) in generalized anxiety disorder, while high doses of the more potent benzodiazepines apparently do work in panic. All of this might well suggest that panic is indeed just a more intense reaction than generalized anxiety disorder. However, one thing has always struck me about panic $v s$ generalized anxiety disorder - the former relates very much more to a disturbance ('catastrophic interpretation') in the processing of internal physiological stimuli while the latter appears to relate much more to a general disturbance ('hypervigilance') in the processing of stimuli from the external world. So, the dysfunction in each case is not with respect to the defensive reaction per se but with earlier stages of information-processing (i.e., interpretation of input as signifying danger/threat when it is not - leading to inappropriate or exaggerated response?). As such, models focussing on adaptive responses of normal animals to an actual/potential threat (virtually all models) may be missing the point. This would argue for the need to very carefully select species and/or strains not only for high levels of 'trait' defensiveness (measured in diverse contexts) but also for the tendency to display such responses to non-threatening as well as threatening events.

Roberto Andreatini: I believe that panic could be a big anxiety. The clear separation between panic and generalized anxiety disorder proposed earlier is less clear today. One important aspect of this separation was the difference in the pharmacological treatment of these disorders: tricyclic antidepressants for panic and benzodiazepines for generalized anxiety disorder. However, recent papers suggest that panic can be treated with benzodiazepines (alprazolam and clonazepam) and generalized anxiety disorder can be treated with tricyclic antidepressants and serotonin reuptake inhibitors (4).

Frederico Graeff: Roberto, there are opposite results with ritanserin, an experi- 
mental 5-HT2 antagonist. Anxiety is relieved, but panic is aggravated.

Francisco Guimarães: The same could be said about d-fenfluramine (5).

Sheila Handley: Buspirone is active in generalized anxiety disorder but it has no significant benefit in panic disorder.

Guy Griebel: I also believe that panic may be a big anxiety attack. The alleviation of panic symptoms usually requires high doses of benzodiazepines which are very effective at low doses against generalized anxiety, perhaps a more moderate type of anxiety disorder $(6,7)$. However, panic may also be something clearly different from generalized anxiety in that the mechanisms involved seem to be different. While there is little doubt that generalized anxiety involves the GABA/benzodiazepine receptor complex, panic involves (in addition?) other neurotransmitters such as serotonin, noradrenaline or even several neuropeptide systems such as cholecystokinin.

Caroline \& Robert Blanchard: Guy, regarding your informative comment, are you saying that benzodiazepines may be regarded as relatively selective antipanic compounds, but only when given chronically at high doses? Puts them right in with other antipanic drugs. Are there any antipanic drugs that work acutely?

Francisco Guimarães: Robert and Caroline, based on data from our public speaking model we suggested that d-fenfluramine could act acutely. Dr. Luiz A. Hetem, here in Brazil, treated successfully some patients with the drug before it was withdrawn from the market.

Roberto Andreatini: I think that in clinical settings the effect of benzodiazepines is faster than that of antidepressive drugs in reducing panic attacks $(8,9)$.

Guy Griebel: Roberto, benzodiazepines may be effective against panic after only a few days of treatment, whereas antidepressants, especially tricyclics, require several weeks to show some reduction in the occur- rence of panic frequency $(9,10)$.

Roberto Andreatini: Yes, I agree with you.

Frederico Graeff: Guy, although benzodiazepines are quicker to improve panic than antidepressants, if you compare to generalized anxiety disorder it seems that you need higher doses of potent compounds and several days.

Guy Griebel: Yes, I agree with you.

Guy Griebel: Robert and Caroline, there is a very rapid tolerance to the sedative action of high doses of benzodiazepines following repeated treatment (11). This leaves us, I believe, with a specific anxiolytic effect (on panic) which shows no development of tolerance.

Caroline \& Robert Blanchard: Guy, I take your point about the benzodiazepines, but how specific is the benzodiazepine effect on panic. Really high benzodiazepine doses will reduce just about anything!

Guy Griebel: Yes, some benzodiazepines (alprazolam, clonazepam, diazepam, clorazepate) are specific antipanic compounds after repeated treatment with high doses (6). On a clinical level, no drug (so far) works specifically (i.e., without producing sedation) against panic after a single administration.

Frederico Graeff: Luiz, I think one important difference between panic and anxiety is that in the latter adrenocorticotropic hormone (ACTH) is not released. I believe you have data showing that electrical stimulation of the dorsal periaqueductal gray matter does not activate the hypothalamus-pituitary-adrenal axis. Am I right?

Luiz Carlos Schenberg: Yes, likewise in panic attacks, neither ACTH nor plasma prolactin levels showed any change following 1 min full-blown freezing and flight behaviors. There seems to be an active inhibition of the hypothalamus-pituitary-adrenal axis, because no one who experienced a panic attack could say that it is not "stressing".

Francisco Guimarães: This lack of 
ACTH increase is really puzzling. If I am not wrong, the hippocampus exerts an inhibitory influence on the paraventricular nucleus. Could it be involved in this effect?

Sheila Handley: Frederico and Luiz - I would like to put in a comment here. I wonder what the baseline values were in your experiments, Luiz? If patients or animals are already stressed, important effects on circulating corticosteroids can be obscured (see Ref. 12). Also, prolonged elevation of circulating corticosteroids is likely to down-regulate the receptors responsible for negative feedback; this is one reason put forward for the hypercortisolemia of depression but we don't know if it occurs in anxiety disorders. The literature on hypothalamus-pituitaryadrenal axis activation in panic attacks is inconsistent (see Refs. 13 and 14) and difficulty of obtaining a satisfactory baseline in panic-disorder patients could be a factor. Nevertheless, acute increases in salivary cortisol have been demonstrated recently during spontaneous panic attacks (13). The circadian trough for circulating corticosteroids, together with the threshold of the stress response is thought to be controlled by mineralocorticoid receptors, probably in the hippocampus, while glucocorticoid receptors have been suggested to govern the extent and duration of the stress-induced rise by controlling the rate of return to baseline through negative feedback (see Refs. 12 and 15). It may be that all prevailing corticosteroid levels above the circadian trough are actually due to ongoing 'microstresses'. We have recently measured afternoon salivary cortisol in a familiar environment in children with attention deficit hyperactivity disorder and found significantly lower levels; we suggest this could be because they are not processing properly the environmental information that would normally lead to a parallel inhibition of behavior and activation of the hypothalamus-pituitary-adrenal axis (16). On a slightly different track, we knocked down the glycocorticoid receptors with an anti- sense oligonucleotide delivered $i c v$ in rats and found a parallel increase in 5-HT2A receptor expression accompanied by an increase in 2,5-dimethoxy-4-iodoamphetamine-induced head-shakes (17). This illustrates the potential importance of the hypothalamus-pituitary-adrenal axis in controlling 5-HT responsiveness but we haven't yet looked at the effect on anxiety.

Caroline \& Robert Blanchard: Luiz, what about autonomic changes in conjunction with lack of hypothalamus-pituitaryadrenal axis arousal, in panic?

Luiz Carlos Schenberg: The autonomic changes of dorsal periaqueductal gray matter-induced flight are quite similar to panic attacks, shallow and fast respiration, tachycardia and hypertension. The shallow fast respiration is very important from a clinical viewpoint.

Caroline \& Robert Blanchard: I am interested in the hypothalamus-pituitary-adrenal axis inhibition or shutdown of panic because we are very consistently getting a similar lack of hypothalamus-pituitary-adrenal axis response to acute stress in socially stressed male rats. They don't, off hand, look like panickers, but there could be some sort of exhaustion effects since they (and panickers) are certainly repeatedly aroused. Do you have any information on the time course of this hypothalamus-pituitary-adrenal axis "inhibition"?

Luiz Carlos Schenberg: Very important results indeed! There are results in the literature, however, with the dominant-subordinate paradigm, showing the opposite - increased ACTH levels!

Caroline \& Robert Blanchard: It may be a matter of time and thresholds... In our models we get this shutdown in both corticosterone and in paraventricular nucleus corticotropic releasing factor expression, in about 25 to $45 \%$ of subordinates, by about 12-14 days of grouping. No trace of this yet in the other subordinates, and they tend to have higher basal levels of corticosterone. 
Luiz Carlos Schenberg: The amygdala seems to have another relationship with hypothalamus-pituitary-adrenal axis.

Padua Carobrez: I don't know for sure if panic is a big anxiety or something else. For me the study of panic has to be divided into two parts; an unconditioned (the panic attack) component and a learned component, that is able to keep the response going on (contextual learning). In my opinion, a reliable animal model for panic should be able to include both parts.

Frederico Graeff: Marcus, I recall that Daniel Vianna's results in your lab showed that electrolytic lesion of the ventrolateral periaqueductal gray matter impaired freezing generated by contextual fear conditioning, like in Fanselow's original observations (18). However, freezing induced by electrical stimulation of the dorsal periaqueductal gray matter remained unchanged. My guess is that despite similar behavioral expression (tense immobility) periaqueductal gray matter freezing relates to the complete immobility seen in panic attacks, in addition to flight.

Marcus Brandão: You are right. Lesion of the ventrolateral periaqueductal gray matter impaired contextual freezing, whereas freezing from dorsal periaqueductal gray matter stimulation was not affected by ventrolateral periaqueductal gray matter lesions. I agree with you that probably dorsolateral periaqueductal gray matter freezing may be related to panic attacks.

Frederico Graeff: Luiz, both of you and Francois Jenck work with stimulation of the dorsal periaqueductal gray matter as a model of panic (19). Are the results with panicolytic and panicogenic drugs compatible?

Luiz Carlos Schenberg: In some sense, yes. Fluoxetine selectively attenuated galloping at low doses $\left(1 \mathrm{mg} \mathrm{kg}^{-1}\right.$ day $^{-1}, 21$ days $)$ only, an important component of shuttle-box escape in Jenck's studies.

Francisco Guimarães: Luiz, in the preceding chat on "Emotion" you told me that acute clomipramine decreased defense thresholds (with dorsal periaqueductal gray matter stimulation). I think an important question is what are the mechanisms of the initial symptom worsening sometimes found with selective serotonin reuptake inhibitors in the treatment of panic. Could this involve a shutdown of 5-HT neurons in the dorsal raphe nucleus and a consequent decrease in 5-HTmediated periaqueductal gray matter inhibition? We have recently finished an investigation studying Fos-like immunoreactivity in stressed (by restraint) and non-stressed rats after acute or chronic (21 days) administration of fluoxetine. A single administration of fluoxetine decreased Fos-like immunoreactivity in the dorsolateral periaqueductal gray matter in stressed rats, but chronic treatment increased it in both stressed and nonstressed animals.

Luiz Carlos Schenberg: No Francisco, clomipramine increased the thresholds with chronic administration (10 $\mathrm{mg} \mathrm{kg}^{-1}$ day $^{-1}, 21$ days). More precisely, clomipramine increased the thresholds of immobility, trotting, galloping, jumping and micturition, but not exophthalmus or defecation.

Frederico Graeff: Luiz, in the chat on "Emotions" you suggested that the medial and central amygdala may be involved in innate and learned fear, respectively. Why?

Luiz Carlos Schenberg: I suggested that the medial amygdala could be involved in innate fear of aversive odors.

Claudio Da Cunha: It is interesting that some innate fears, and also anxiety respond well to benzodiazepines and the highest density of benzodiazepine receptors is in the basolateral amygdala (20).

Frederico Graeff: Let us switch to 5HT. Telma Andrade and Hélio Zangrossi Jr. got nice results showing that electrolytic lesion of the median raphe nucleus impaired both inhibitory avoidance (learned fear) and one-way escape (unlearned fear) in the rat elevated T-maze test. 5,7-DHT lesion impaired avoidance, but not escape. Microinjection of 8-OH-DPAT into the median raphe 
nucleus had the same effect as the neurotoxic lesion, which was prevented by local administration of a 5-HT1A receptor antagonist (WAY 100635). These results suggest that 5-HT neurons of the median raphe nucleus regulate learned fear, and non-serotonergic neurons regulate innate fear.

Francisco Guimarães: One important point in relation to the role of 5-HT in panic and a possible inhibition of periaqueductal gray matter is what happens during a flight reaction. Recently, Maione and colleagues (21) showed that N-methyl-D-aspartate (NMDA) injection into the periaqueductal gray matter can decrease 5-HT concentration in this region, whereas bicuculline increased it. To explain these results it is proposed that GABAergic neurons in the periaqueductal gray matter project to the dorsal raphe nucleus. So, we may suppose that during a proximal defense reaction glutamatergic input to the periaqueductal gray matter would command flight and, at the same time, would decrease the inhibitory 5-HT input. Could you comment on this possibility?

Frederico Graeff: These results are enlightening. We suggested a 5-HT inhibitory input to the dorsal periaqueductal gray matter coming from the amygdala (22). These results point to an alternative feedback mechanism.

Marcus Brandão: What about moving to the second point of our chat? "What aspects of anxiety does an animal model of anxiety model?"

Francois Jenck: What aspects of "anxiety" do animal models model? Basic statements: 1) Animals, like humans, express different kinds of fear/anxiety in response to different environmental conditions (i.e., acute vs chronic stress, spontaneous vs conditioned responses, etc.). 2) Only restricted aspects of human psychopathology can be explored and simulated using animal models. As many others, we propose that a few fundamental rules need to be followed: 1) A complete neurobiological evaluation of any anti-anxiety effect requires that effects be examined in several paradigms. 2) The continuum existing clinically between anxiety and depressive disorders must be taken into consideration. 3) The best animal models must be evaluated and selected for their face validity, predictive validity, and construct validity. We have obtained pharmacological evidence for preferentially relating some animal models to specific anxiety disorders in man. In addition, many contradictions about the role of neurotransmitter systems in anxiety can probably be explained by their anatomical, pharmacological and functional heterogeneity. Clinical and preclinical data are compatible with the hypothesis that different neurotransmitters/modulators have distinct and opposite roles in modulating different kinds of anxiety in different brain regions. Deakin and Graeff theory (2): The opposite patterns of results observed with 5-HT receptor agonists and antagonists in different models are not mutually exclusive but rather suggest that 5-HT receptor subtypes exert an elaborate control over different types of anxiety. For instance, 5-HT2C neurotransmission selectively modulates specific kinds of anxiety generated by different animal models (23). This is in line with the Deakin and Graeff theory (2) on the complex action of 5-HT on the neural mechanism of anxiety where 5HT is hypothesized and found to either facilitate or inhibit different kinds of fear in different brain regions. Different anxiety states in animals or man probably recruit and involve different brain regions and receptor types, either alone or in combination, hence with different symptomatic outcomes. In that respect, the use of nonselective probes such as m-CPP is likely to generate contaminated results and cannot be applied to the elucidation of the physiological machinery involved.

Guy Griebel: The easiest way to answer this question is to look at the pharmacological data obtained with anxiety models. Conflict models which are mostly sensitive to 
benzodiazepines, drugs used to treat generalized anxiety, may probably only model aspects of this condition. Tests that rely on exploratory behaviors are much more complex as they are sensitive to all kind of psychoactive drugs (some of them are not even active against any anxiety disorder). This is probably because different motivations can lead to the same behavioral outcome in exploration models (i.e., an increase in time spent in an aversive area). Factor analysis has clearly shown that behaviors in exploration models (notably the elevated plus-maze) relate to different aspects of emotional processes. Benzodiazepines are very effective in these tests. As such, exploration tests model predominantly certain aspects of generalized anxiety. Do exploration models also model panic? I have some doubts, as 5-HT reuptake inhibitors, which are the mainstay of drug treatment in this condition, are generally not active in these tests. What about phobias? The problem with this condition is that there are not that many effective pharmacological treatments.

Frederico Graeff: Guy, then, models of phobia should be sensitive to exposure?

Guy Griebel: I'm not sure about that, Frederico. Phobias are more cognitive-oriented anxiety disorders, which are very difficult to model in animals. To illustrate this idea, propranolol, which is among the most effective drug treatments against social phobia, is inactive in all anxiety models (24).

Roberto Andreatini: Which phobia? Social, agoraphobia, or simple phobia? Social phobia has a moderate response to monoamine oxidase inhibitors (e.g., phenelzine). On the other hand, simple phobia is unlikely to show any improvement with pharmacological treatment.

José Roberto Leite: Frederico, if we consider learned fear, exposure is a very effective procedure. For instance, in passive avoidance, where we exposed the animals to the situation without punishment, the fear of a dark place is quickly extinguished. In the elevated plus-maze, on the other hand, exposure to open arms is not effective to modify the avoidance behavior of the open arm.

Claudio Da Cunha: In passive avoidance, animals do not naturally fear the dark compartment. They learn to fear it after the shock. If the unconditioned stimulus (shock) remains present they will continue to fear it.

Luiz Carlos Schenberg: Guy, may be conditioned fear and unconditioned fear have different pharmacological profiles, like anxiety and panic. In our model, threshold logistic analysis, fluoxetine inhibited galloping only, but not the other dorsal periaqueductal gray matter-induced defensive behavior.

Marcus Brandão: Luiz, getting back to the problem of physiological and pathological panic. Considering that we work with animal models, how can we clearly distinguish between them in animals? Can we specify these conditions before conducting any particular experiments?

Luiz Carlos Schenberg: No, we can't.

Frederico Graeff: Marcus, animals are obviously not in panic in many animal models, like the defense test battery or escape in the elevated T-maze. What may be claimed is that we are dealing with mechanisms underlying adaptive behavior that are supposed to go wrong in panic disorder. In this respect, Maria Cristina L. Silveira carried out a c-Fos study in rats on the elevated T-maze. Performance of the avoidance task (withdrawal from the enclosed arm) increased Fos-like immunoreactivity in the medial nucleus of the amygdala, paraventricular nucleus of the thalamus, anterior hypothalamic nucleus and median raphe nucleus. In contrast, performance of escape (from the open arm) enhanced Fos-like immunoreactivity in the dorsal periaqueductal gray matter. Both behavioral tasks increased Fos-like immunoreactivity in the dorsomedial nucleus of the hypothalamus. Therefore, inhibitory avoidance and one-way escape activated different sets of brain structures, respectively. This evidence supports the original hypothesis that 
two types of fear/anxiety are generated in the elevated T-maze, learned and unlearned, respectively.

Frederico Graeff: Guy, do you think we have a good animal model of panic?

Guy Griebel: There are several models that claim to model panic (dorsal periaqueductal gray matter stimulation, T-maze, mouse defense test battery...), mainly based on pharmacological data (25). My opinion is that a model of panic is predictive of an antipanic-like activity or may be considered as reliable for modeling this condition if different laboratories using the same model have found similar results. That is not really the case at the present time because nobody is using the same panic test.

Sheila Handley: In animal models we are only able to model the behavioral output. I think we need a much tighter definition of panic, otherwise we can make of it what we like. For instance, is freezing a symptom of panic or not? Freezing is very rare in humans and it is not listed as a symptom of panic disorder in DSM IV ${ }^{1}$ or ICD- $10^{2}$. On the other hand, the desire to flee, or actual flight (...an exit, usually hurried, from wherever he or she may be (ICD-10)) are common in human panic and can sometimes lead to disastrous attempts to escape. On that basis, the periaqueductal gray matter stimulation and other models that elicit flight behavior all have face-validity.

Caroline \& Robert Blanchard: One way might be through the use of agents that can produce panic in people. Cocaine is working very well in our rats, in eliciting a high level panic-like flight response, which could then be used in conjunction with other manipulations. Haven't used sodium lactate yet, but plan to. Another approach (already underway) is to try to understand the biology of the defense systems, with a second tactic of trying to experimentally link specific aspects of defense to particular defense-related psychopathologies.

Frederico Graeff: José Roberto, you have worked on pentylenetetrazole and anxiety. Pentylenetetrazole induces panic-like states in humans. Before falling into fits, rats run wildly - as if in panic - in the soundinduced epilepsy model. Are panic and convulsions related?

José Roberto Leite: I believe that clonic convulsions induced by pentylenetetrazole could be a model for panic. If we consider the descriptions of the behavioral effect in humans this should be an interesting animal model. We do not forget about the benzodiazepine specific antagonism of the effects of pentylenetetrazole. If we consider the clinical point of view, I think that this should be a limitation of the model.

Padua Carobrez: Pentylenetetrazole (15 and $30 \mathrm{mg} / \mathrm{kg}$ )-induced anxiety, measured in the plus-maze, disappeared after NMDA/ glycine antagonism into the dorsal periaqueductal gray matter (26).

Frederico Graeff: Sorry, but it is time to end. I am thankful to Claudio Da Cunha for organizing this chat, and to all participants. It was very stimulating. Best wishes to everyone.

Claudio Da Cunha: In the name of the Brazilian Society of Neuroscience and Behavior I would like to thank you all and say that it was a pleasure to be with you in this chat.

\section{Acknowledgments}

The SBNeC organizers are grateful to Hidevaldo Machado and Roberto Andreatini for the help with the organization of the chat and to Simone Alencar for technical assistance during the chat. CNPq-Prossiga is also acknowledged for providing the chat web room. 


\section{References}

1. Taylor J G (1962). The Behavioral Basis of Perception. Yale University Press, New York.

2. Deakin J FW \& Graeff FG (1991). 5-HT and mechanisms of defense. J oumal of Psychopharmacology, 5: 305-315.

3. Graeff FG, Guimarães FS, De Andrade TGCS \& Deakin J FW (1996). Role of 5-HT in stress, anxiety and depression. Pharmacology, Biochemistry and Behavior, 54: 129-141.

4. J anicak PG, Davis J M, Preskorn SH \& Ayd J r FJ (1997). Principles and Practice of Psychopharmacotherapy. 2nd edn. Williams \& Wilkins, Baltimore.

5. Hetem LA, De Souza CJ , Guimarães FS, Zuardi AW \& Graeff FG (1996). Effect of d-fenfluramine on human experimental anxiety. Psychopharmacology, 127: 276282.

6. Ballenger J C (1990). Efficacy of benzodiazepines in panic disorder and agoraphobia. J ournal of Psychiatric Research, 24 (Suppl 2): 15-25.

7. Bradwejn J (1993). Benzodiazepines for the treatment of panic disorder and generalized anxiety disorder: Clinical issues and future directions. Canadian J ournal of Psychiatry, 38 (Suppl 4): S109-S113.

8. Tesar GE, Rosenbaum J F, Pollack MH, Otto MW, Sachs GS, Herman J B, Cohen LS \& Spier SA (1991). Double-blind, placebo-controlled comparison of clonazepam and alprazolam for panic disorder. J ournal of Clinical Psychiatry, 52: 69-76.

9. Liebowitz MR (1989). Tricyclic antidepressants and monoamine oxidase inhibitors in the treatment of panic disorder: Brief review. Psychopharmacology Bulletin, 25: 17-20.

10. J ohnson MR, Lydiard RB \& Ballenger J C (1995). Panic disorder: Pathophysiology and drug treatment. Drugs, 49: 328-344.
11. Hutchinson MA, Smith $P F \&$ Darlington $\mathrm{CL}$ (1996). The behavioural and neuronal effects of the chronic administration of benzodiazepine anxiolytic and hypnotic drugs. Progress in Neurobiology, 49: 7397.

12. J acobson L \& Sapolsky R (1991). The role of the hippocampus in feedback regulation of the hypothalamic-pituitary-adrenocortical axis. Endocrine Reviews, 12: 118134.

13. Bandelow B, Wedekind D, Pauls J , Brooks A, Hajak G \& Ruther E (2000). Salivary cortisol in panic attacks. American J ournal of Psychiatry, 157: 454-456.

14. Sinha SS, Coplan J D, Pine DS, Martinez J A, Klein DF \& Gorman J M (1999). Panic induced by carbon dioxide inhalation and lack of pituitary-adrenal axis activation. Psychiatry Research, 86: 93-98.

15. Heuser I (1998). The hypothalamic-pituitary-adrenal system in depression. Pharmacopsychiatry, 31: 10-13.

16. Kariyawasam $\mathrm{SH}$, Zaw $\mathrm{F} \&$ Handley $\mathrm{SL}$ (2000). Low salivary cortisol in children with attention deficit hyperactivity disorder/oppositional defiant disorder. Biological Psychiatry (in press)

17. Islam A, Thompson KSJ, Akhtar $S \&$ Handley SL (2000). Antisense oligodeoxynucleotide to rat glucocorticoid receptor, given icv in polymer microspheres, reduces glucocorticoid- and increases 5HT2A-receptor binding. British J ournal of Pharmacology, 129 (Suppl S): 244P.

18. Fanselow MS (1991). The midbrain periaqueductal gray as a coordinator of action in response to fear and anxiety. In: Depaulis A \& Bandler R (Editors), The Midbrain Periaqueductal Gray Matter: Functional, Anatomical and Immunohistochemical Organization. Plenum Publishing Corp., New York, 151-173.
19. Graeff FG (1991). Neurotransmitters in the dorsal periaqueductal gray and animal models of panic anxiety. In: Briley $M \&$ File SE (Editors), New Concepts in Anxiety. MacMillan Press, London, 288-312.

20. Niehoff DL \& Kuhar MJ (1983). Multiple benzodiazepine receptors: autoradiographic localization in normal human amygdala. Brain Research, 16: 237-245.

21. Maione S, Palazzo E, De Novellis V, Stella L, LeysaJ \& Rossi F (1998). Metabotropic glutamate receptors modulate serotonin release in the rat periaqueductal gray matter. Naunyn-Schmiedeberg's Archives of Pharmacology, 358: 411-417.

22. Graeff FG, Silveira MCL, Nogueira RL, Audi EA \& De Oliveira RMW (1993). Role of the amygdala and periaqueductal gray in anxiety and panic. Behavioural Brain Research, 58: 123-132.

23. J enck F, Boes M, Wichmann J , Stadler $H$, Martin J R \& Moreau J -L (1998). The role of $5 \mathrm{HT} 2 \mathrm{C}$ receptors in affective disorders. Expert Opinion on Investigational Drugs, 7: 1587-1599.

24. Griebel G (1995). 5-Hydroxytryptamineinteracting drugs in animal models of anxiety disorders: More than 30 years of research. Pharmacology and Therapeutics, 65: 319-395.

25. Griebel G, Blanchard DC \& Blanchard RJ (1996). Predator-elicited flight responses in Swiss-Webster mice: An experimental model of panic attacks. Progress in NeuroPsychopharmacology and Biological Psychiatry, 20: 185-205.

26. De Souza MM , Schenberg LC \& Carobrez AP (1998). NMDA-coupled periaqueductal gray glycine receptors modulate anxioselective drug effects on plus-maze performance. Behavioural Brain Research, 90: 157-165. 